\title{
La troisième statue de Sésostris III au musée de Khartoum
}

Par

\section{Claude Obsomer}

Université de Namur, Université catholique de Louvain

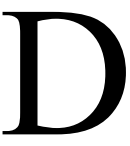

ans l'article que j'ai consacré aux stèles nubiennes de l'an 16 de Sésostris III, dans un précédent numéro du $B A B E L A O$, il fut question brièvement de trois statues de ce roi conservées au musée de Khartoum ${ }^{1}$. Deux d'entre elles étaient bien identifiées, bien que leurs inscriptions fussent encore inédites. Mais ce qui fut écrit de la troisième est erroné, comme m'ont permis de le constater une visite personnelle du musée effectuée en février 2019 et la lecture de l'article récent que Vivian Davies a consacré à ces statues ${ }^{2}$. Son article propose pour la première fois une étude des inscriptions de celles-ci, accompagnée de photographies.

La première statue (Khartoum $\mathrm{n}^{\circ} 447$ ) est visible dans le jardin du musée, dans le hangar qui abrite le temple de Touthmosis III à Semna-Ouest, dans lequel elle avait été découverte ${ }^{3}$. La deuxième (Khartoum $n^{\circ} 452$ ) vient de la forteresse d'Ouronarti ${ }^{4}$ : elle est exposée dans le

\footnotetext{
${ }^{1}$ Cl. OBsomer, «Sésostris III et la frontière de Semna: une analyse des stèles nubiennes de l'an 16 », BABELAO, 6, 2017, p. 21-22.

${ }^{2}$ W.V. DAVIES, «Statues of Senwosret III in the Sudan National Museum, Khartoum », dans Nathalie FAVRY et alii, Du Sinaï au Soudan, itinéraires d'une égyptologue. Mélanges offerts au professeur Dominique Valbelle, Paris, 2017, p. 75-85.

${ }^{3}$ DAVIES, p. 77-78, fig. 6-12. « Sandstone. Height: about $123 \mathrm{~cm}$; width, about $45 \mathrm{~cm}$; depth, about $76 \mathrm{~cm} »$.

${ }^{4}$ DAVIES, p. 75-77, fig. 1-5. « Hard sandstone (?). Dimensions: Ht: 1.09 m; W: 43 cm; Depth: $85 \mathrm{~cm}$ ».
} 
musée lui-même, au rez-de-chaussée, à droite de la stèle d'Ouronarti (Khartoum $\mathrm{n}^{\circ}$ 451) dont j'ai proposé une nouvelle copie autographe en $2017^{5}$. Ces deux statues semblent postérieures au règne de Sésostris III et ont pu être placées au Nouvel Empire dans deux forteresses où le roi était vénéré comme une divinité. Quant à la troisième statue, ce n'est pas celle qui est exposée au musée quelques mètres à droite de la précédente, car les inscriptions de celles-ci mentionnent Aménophis I ${ }^{\mathrm{er}}$ de la XVIII ${ }^{\mathrm{e}}$ dynastie $^{6}$.

La troisième statue de Sésostris III est, en réalité, un fragment de statue royale en granite rose découvert à Semna et conservé dans les réserves du musée (Khartoum $n^{\circ} 448$ ) : Davies en publie des photographies qui permettent d'en découvrir les inscriptions au nom de Sésostris $\mathrm{III}^{7}$. Le roi est figuré agenouillé, mais son torse et le haut de son corps sont manquants. L'inscription latérale droite précise que le roi est « aimé de [...] Éléphantine » (mry [...] 3bw), ce qui amène Davies à supposer la mention de la déesse Satet/Satis, maîtresse d'Éléphantine ${ }^{8}$. D'autres inscriptions attestent à plusieurs reprises le début de la séquence «aimé de ... (divinité) », et pouvaient, selon Davies, mentionner d'autres dieux, comme Dédoun, Khnoum et Anouqet ${ }^{9}$.

Deux autres fragments de granite rose ont été découverts à Semna par Reisner en 1924 (Boston MFA 24.1764) ${ }^{10}$. Le premier fragment offre la partie droite du visage caractéristique de Sésostris III, tandis que le second est un élément de pagne plissé. Ces deux éléments de granite rose pourraient-ils appartenir à la statue agenouillée conservée dans les réserves du musée de Khartoum ? Dans l'affirmative, il pourrait bien s'agir de la statue placée à Semna par Sésostris III lui-même, suivant les indications des stèles de l'an 16: "Voici donc que Ma Majesté a fait faire une représentation / statue (twt) de Ma Majesté sur cette frontière que Ma Majesté a établie, afin que vous soyez fermes à cause d'elle et afin que vous luttiez pour elle ».

\footnotetext{
${ }^{5}$ OBSOMER, p. 38.

${ }^{6}$ La statue est renseignée comme une « statue de Sésostris III » sur un plan du musée de Khartoum accessible en ligne, et la photographie dont je disposais en 2017 ne permettait pas de lire ses inscriptions.

${ }^{7}$ DAVIES, p. 79-80, fig. 13-17. « Red granite. Max. surviving height about $38 \mathrm{~cm}$; max. depth, about $45 \mathrm{~cm}$; max width, about $30 \mathrm{~cm}$; height of pedestal, $16 \mathrm{~cm} »$.

${ }^{8}$ DAVIES, p. 80.

${ }^{9}$ DAVIES, p. 81.

${ }^{10}$ La « quatrième statue » mentionnée dans mon article.
} 